Check for updates

Cite this: Mol. Omics, 2021, 17,641

DOI: 10.1039/d1mo90014e

rsc.li/molomics

\section{Correction: The pan-cancer analysis of the two types of uterine cancer uncovered clinical and prognostic associations with m6A RNA methylation regulators}

\author{
Zhilin Zou, ${ }^{\text {ac }}$ Shuguang Zhou, ${ }^{\mathrm{b}}$ Guosheng Liang, ${ }^{\mathrm{C}}$ Zhenye Tang, $^{\mathrm{C}} \mathrm{Kai} \mathrm{Li}^{\mathrm{c}}$ \\ Shuzhen Tan, ${ }^{c}$ Xiangsheng Zhang ${ }^{c}$ and Xiao Zhu (D)*acd
}

Correction for 'The pan-cancer analysis of the two types of uterine cancer uncovered clinical and prognostic associations with m6A RNA methylation regulators' by Zhilin Zou et al., Mol. Omics, 2021, 17, 438-453, DOI: 10.1039/d0mo00113a.

The authors apologise that the addresses and contact details for the authors Zhilin Zou, Guosheng Liang, Zhenye Tang, Kai Li, Shuzhen Tan, Xiangsheng Zhang and Xiao Zhu were given incorrectly previously. The correct addresses are shown here.

The authors regret that there was an error in the funding information. "This work was supported partly by National Natural Science Foundation of China (81541153); Guangdong Science and Technology Department (2016B030309002 and 2019B090905011); The Fund of Southern Marine Science and Engineering Guangdong Laboratory (Zhanjiang) (ZJW-2019-007); and The Public Service Platform of South China Sea for R\&D Marine Biomedicine Resources (GDMUK201808). The funders had no role in the design of the study; the collection, analysis, and interpretation of the data; the writing of the manuscript; and the decision to submit the manuscript for publication." Should be replaced by "This work was supported partly by National Natural Science Foundation of China (81541153 and 81972366); Guangdong Science and Technology Department (2016B030309002 and 2019B090905011); The Fund of Southern Marine Science and Engineering Guangdong Laboratory (Zhanjiang) (ZJW-2019-007); Guangdong Key Laboratory funds of Systems Biology and Synthetic Biology for Urogenital Tumors (2017B030301015); and The Public Service Platform of South China Sea for R\&D Marine Biomedicine Resources (GDMUK201808). The funders had no role in the design of the study; the collection, analysis, and interpretation of the data; the writing of the manuscript; and the decision to submit the manuscript for publication."

The Royal Society of Chemistry apologises for these errors and any consequent inconvenience to authors and readers.

\footnotetext{
${ }^{a}$ Anhui Clinical and Preclinical Key Laboratory of Respiratory Disease, Department of Respiratory Disease, The First Affiliated Hospital of Bengbu Medical College, Bengbu 233004, China.E-mail: xzhu@gdmu.edu.cn, bioxzhu@yahoo.com

${ }^{b}$ Department of Gynecology, Maternity and Child Healthcare Hospital of Anhui Medical University, Hefei 230001, China

${ }^{c}$ Guangdong Key Laboratory for Research and Development of Natural Drugs, The Marine Biomedical Research Institute, Guangdong Medical University, Zhanjiang 524023, China

${ }^{d}$ Guangdong Provincial Key Laboratory of Systems Biology and Synthetic Biology for Urogenital Tumors, Department of Urology, The First Affiliated Hospital of Shenzhen University, Shenzhen Second People's Hospital (Shenzhen Institute of Translational Medicine), Shenzhen 518035, China
} 